\title{
Synthesis of some 4-bromo-1-naphthyl chalcones using silica-sulfuric acid reagent under solvent free conditions
}

\author{
Ganesamoorthy Thirunarayanan $^{\text {*a }}$ and Ganesan Vanangamudi ${ }^{\text {b }}$ \\ ${ }^{a}$ Department of Chemistry, Annamalai University, Annamalainagar-608 002, India \\ ${ }^{b}$ Postgraduate Department of Chemistry, Govt. Arts College, Chidambaram-608 102, India \\ E-mail:_rgtnarayanan@yahoo.com
}

\begin{abstract}
A series of $\alpha, \beta$-unsaturated ketones derived form 4-bromo-1-naphthyl ketones with various substituted benzaldehydes under solvent free condition using silica-sulfuric acid as a reagent in an oven. The catalyst silica is reusable and the yields of chalcones are more than $90 \%$. These chalcones are characterized by physical constants and spectral data. No decrease in the yield was observed, demonstrating that silica-sulfuric acid can be re-used in crossed-aldol condensation reaction without environmental discharge.
\end{abstract}

Keywords: Styryl-4-bromo-1-naphthyl ketones, silica-sulfuric acid, crossed-aldol reaction, solvent free synthesis, environmentally begin reaction

\section{Introduction}

Recently there has been tremendous interest in the application of solvent-free methods ${ }^{1}$ in organic reactions such as the Claisen-Schmidt, ${ }^{2}$ Knoevenagel, ${ }^{3}$ Aldol, ${ }^{4}$ and Crossed-Aldol ${ }^{5}$ reactions which are employed for the synthesis of carbonyl compounds due to the operational simplicity, easier work-up, better yield and eco-friendly nature. Among these reactions, the aldol condensation is useful for the formation of carbon-carbon bonds in many kinds of carbonyl compounds, ${ }^{6}$ due to the importance of the methylene structural unit, which is found in many naturally occurring compounds, antibiotics, and the use of cyclic and acyclic ketones as precursors for the synthesis of pyrimidine derivatives. ${ }^{7}$ The basic skeleton of chalcones figures widely in natural products which are known to have multi-pronged activity. ${ }^{8}$ Many of the chalcones are used as agrochemicals and drugs. ${ }^{9}$ The condensation of ketones with aldehydes is of special interest, and the crossed-aldol condensation is an effective pathway for those preparations. However, traditional acid-base catalyzed reactions suffer from the reverse reaction ${ }^{10}$ and self-condensation of the starting molecules. ${ }^{11}$ 
Many reagents and coordination complexes of $\mathrm{Mn}(\mathrm{II}), \mathrm{Fe}(\mathrm{II}), \mathrm{Co}(\mathrm{II}), \mathrm{Ni}(\mathrm{II}), \mathrm{Cu}(\mathrm{II})$ and $\mathrm{Zn}(\mathrm{II})$ ions with various ligands have been employed for aldol condensation. ${ }^{12}$ Metal salts of $\mathrm{Cp}_{2} \mathrm{ZrH}_{2}$ are used for condensation of cycloalkanones. ${ }^{13} \mathrm{KF}-\mathrm{Al}_{2} \mathrm{O}_{3}$ and bis- $(p$-methoxy phenyl) tellurides are have been used for crossed condensation under microwave irradiation. ${ }^{14}$ Anhydrous $\mathrm{RuCl}_{3}$ and $\mathrm{TiCl}_{3}\left(\mathrm{SO}_{3} \mathrm{CF}_{3}\right)$ have also been applied for aldol condensation reactions under solvent-free conditions. ${ }^{15}$ Now more attention has been paid to synthesis of acyclic and cyclic chalcones by chemists. ${ }^{16}$ Balakrishna Kalluraya ${ }^{17}$ et al. reported that they obtained 60 $70 \%$ yield of sydnone chalcones under solvent free condition by aldol condensation reaction by grinding of ketones and aldehydes with sodium hydroxide. Silica-sulfuric acid is used as a versatile and stable solid acid catalyst for organic synthesis. ${ }^{18}$ The authors wish to report an efficient and selective method for condensation of 4-bromo-1-naphthyl ketones with various substituted benzaldehydes under solvent free conditions using silica-sulfuric acid as a reagent in an oven to yields the respective E-2-propen-1-ones. The promoting effect of silica - sulfuric acid in their reaction showed good performance and is proved by obtaining higher percentage of yields. The product was isolated and the remaining catalyst was washed and reused with fresh substrate for further reactions. No decrease in the yield was observed, demonstrating that silicasulfuric acid can be reused in crossed-aldol condensation reaction without environmental discharge.

\section{Experimental Section}

General Procedures. All chemicals and Analytical Grade solvents were purchased from EMerck chemical company. Melting points of all chalcones were determined in open glass capillaries on Mettler FP51 melting point apparatus and are uncorrected. Infrared spectra $(\mathrm{KBr}$, $4000-400 \mathrm{~cm}^{-1}$ ) were recorded on a Perkin-Elmer Fourier transform spectrophotometer. The nuclear magnetic resonance spectra, both ${ }^{1} \mathrm{H}$ - and ${ }^{13} \mathrm{C}$-, of chalcones were recorded using UNITYPLUS-300 "KIBSIPS" 300MHz spectrometer. Microanalyses of all chalcones were performed using a Perkin-Elmer 240C Analyzer.

Preparation of silica-sulfuric acid. Silica-sulfuric acid was prepared according to the reported ${ }^{18}$ method and characterized by comparison of their spectral data (IR and NMR) and physical properties with those reported in the literature. ${ }^{13,16 a, 19}$

\section{General procedure for crossed-aldol condensation of aromatic ketones with benzaldehydes}

4-Bromo-1-naphthyl ketones (2 mmol), m-and p-substituted benzaldehydes(4.2 mmol) and silica-sulfuric acid( $1.5 \mathrm{~g}$ equal to $4 \mathrm{mmol}$ of $\mathrm{H}^{+}$) were mixed thoroughly, placed in a glass tube and capped (Scheme-1). The mixture was heated in an oven at $80^{\circ} \mathrm{C}$ for $2-3.5 \mathrm{~h}$. After complete conversion of the ketones as monitored by TLC, the mixture was cooled to room temperature. Dichloromethane $(20-30 \mathrm{ml})$ was added and heated for 3-5 minutes. The reagent was removed by filtration. The filtrate was concentrated and the solid residue was recrystallized from ethanol to afford the pure products as pale yellow glittering solid. The catalyst was recycled by washing the 
solid reagent remained on the filter by ethyl acetate $(20 \mathrm{ml})$ followed by drying in an oven at $50^{\circ} \mathrm{C}$ for $2 \mathrm{~h}$. and can be reusable for another reaction run. Based on Hays and Timmons ${ }^{20,21}$ infrared carbonyl-stretching frequencies of s-cis and s-trans conformers are assigned. The NMR chemical shifts (ppm) of ethylene $\alpha, \beta$ protons and carbons are assigned based on reported in earlier literature ${ }^{22-27}$. The characterization data of all chalcones are summarized.

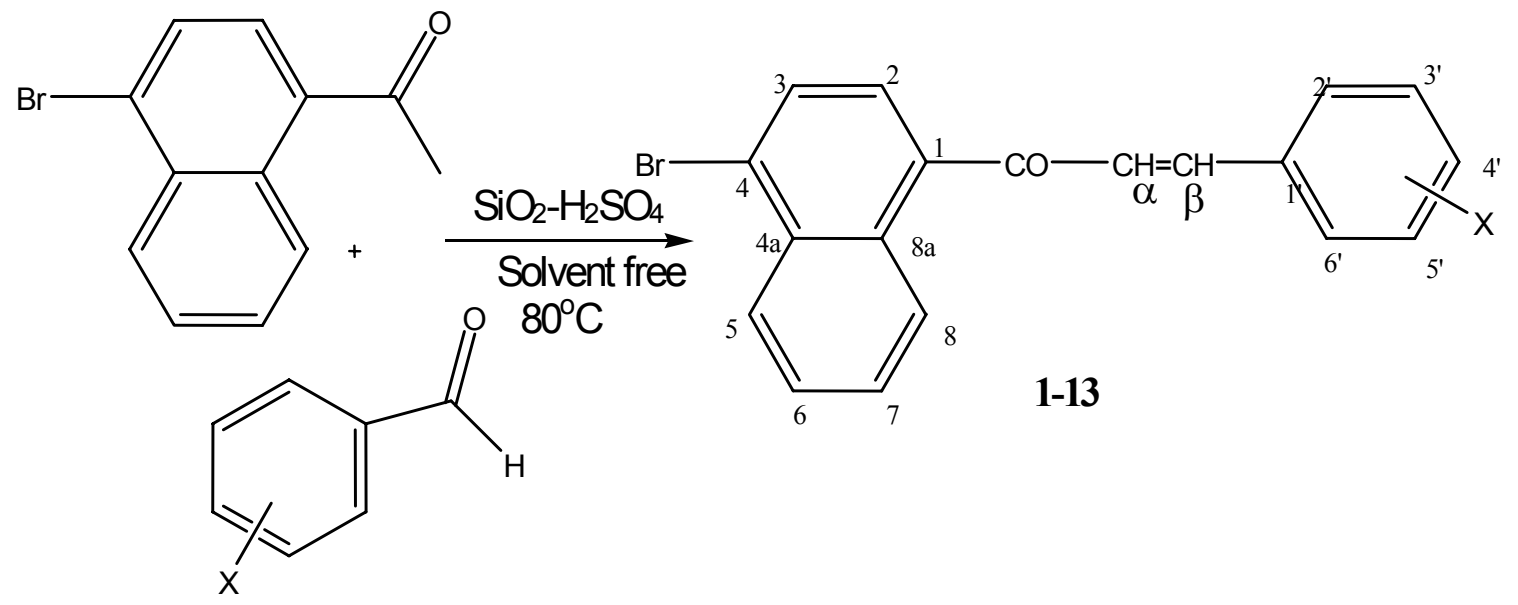

Where $\mathrm{X}=\mathrm{H}, m-\mathrm{NH}_{2}, p-\mathrm{NH}_{2}, m-\mathrm{Br}, m-\mathrm{Cl}, p-\mathrm{Cl}, p-\mathrm{N}\left(\mathrm{CH}_{3}\right)_{2}$ $p-\mathrm{OH}, p-\mathrm{OCH}_{3}, p-\mathrm{CH}_{3}, o-\mathrm{NO}_{2}, m-\mathrm{NO}_{2}, p-\mathrm{NO}_{2}$

\section{Scheme 1}

(2E)-1-(4-Bromo-1-naphthyl)-3-phenyl-2-propen-1-one (1). Yield: 96\%, m.p. 103-104 ${ }^{\circ}$; IR $\left(\mathrm{KBr}, \mathrm{cm}^{-1}\right): v=1687$ (CO s-cis), 1657 (CO s-trans), 984(CH=CH). ${ }^{1} \mathrm{H} \mathrm{NMR}\left(\mathrm{CDCl}_{3}, \mathrm{ppm}\right): \delta=$ $8.25(\mathrm{~d}, 1 \mathrm{H} \alpha), 8.36(\mathrm{~d}, 1 \mathrm{H} \beta), 7.19-7.86(\mathrm{~m}, 11 \mathrm{H} \mathrm{Ar}-\mathrm{H}) .{ }^{13} \mathrm{C}$ NMR $\left(\mathrm{CDCl}_{3}, \mathrm{ppm}\right): \delta=122.85$ $\left(\mathrm{C}_{\alpha}\right) 144.76\left(\mathrm{C}_{\beta}\right), 192.09(\mathrm{CO}), 136.95\left(\mathrm{C}_{1}\right), 129.82\left(\mathrm{C}_{2}\right), 129.91\left(\mathrm{C}_{3}\right), 128.22\left(\mathrm{C}_{4}\right), 129.91\left(\mathrm{C}_{5}\right)$, $126.44\left(\mathrm{C}_{6}\right), 127.30\left(\mathrm{C}_{7}\right), 125.82\left(\mathrm{C}_{8}\right), 133.00\left(\mathrm{C}_{4 \mathrm{a}}\right), 133.74\left(\mathrm{C}_{8 \mathrm{a}}\right), 137.20\left(\mathrm{C}_{1}{ }^{\prime}\right), 126.00\left(\mathrm{C}_{2}^{\prime}, \mathrm{C}_{6}{ }^{\prime}\right)$, 128.66 $\left(\mathrm{C}_{3}^{\prime}, \mathrm{C}_{5}{ }^{\prime}\right), 138.22\left(\mathrm{C}_{4}{ }^{\prime}\right)$. Anal. Calcd for $\mathrm{C}_{19} \mathrm{H}_{13} \mathrm{OBr}(337.20)$ : $\mathrm{C}, 67.67 ; \mathrm{H}, 3.89$. Found: $\mathrm{C}$, $67.59 ; \mathrm{H} 3.82$.

(2E)-1-(4-Bromo-1-naphthyl)-3-(3-aminophenyl)-2-propen-1-one (2). Yield: 92\%, m.p. 72$73^{\circ} \mathrm{C}$; IR $\left(\mathrm{KBr}, \mathrm{cm}^{-1}\right): v=1677\left(\mathrm{CO}\right.$ s-cis), $1644(\mathrm{CO}$ s-trans $), 996(\mathrm{CH}=\mathrm{CH}), 3623\left(-\mathrm{NH}_{2}\right) .{ }^{1} \mathrm{H}$ NMR $\left(\mathrm{CDCl}_{3}, \mathrm{ppm}\right): \delta=8.25(\mathrm{~d}, 1 \mathrm{H} \alpha), 8.31(\mathrm{~d}, 1 \mathrm{H} \beta), 7.31-8.51(\mathrm{~m}, 10 \mathrm{H} \mathrm{Ar}-\mathrm{H}), 4.62(\mathrm{~s}, 2 \mathrm{H}-$ $\left.\mathrm{NH}_{2}\right) .{ }^{13} \mathrm{C} \mathrm{NMR}\left(\mathrm{CDCl}_{3}, \mathrm{ppm}\right): \delta=120.201\left(\mathrm{C}_{\alpha}\right) 143.34\left(\mathrm{C}_{\beta}\right), 192.40(\mathrm{CO}), 136.42\left(\mathrm{C}_{1}\right), 126.00$ $\left(\mathrm{C}_{2}\right), 128.98\left(\mathrm{C}_{3}\right), 127.41\left(\mathrm{C}_{4}\right), 128.42\left(\mathrm{C}_{5}\right), 126.32\left(\mathrm{C}_{6}\right), 128.30\left(\mathrm{C}_{7}\right), 127.82\left(\mathrm{C}_{8}\right), 133.74\left(\mathrm{C}_{4 \mathrm{a}}\right)$, $133.74\left(\mathrm{C}_{8 \mathrm{a}}\right), 132.45\left(\mathrm{C}_{1}{ }^{\prime}\right), 113.20\left(\mathrm{C}_{2}{ }^{\prime}\right), 147.21\left(\mathrm{C}_{3}{ }^{\prime}\right), 115.73\left(\mathrm{C}^{\prime}{ }^{\prime}\right), 130.17\left(\mathrm{C}_{5}{ }^{\prime}\right), 115.73\left(\mathrm{C}_{6}{ }^{\prime}\right)$. Anal. Calcd for $\mathrm{C}_{19} \mathrm{H}_{14} \mathrm{BrNO}$ (337.20): C, 64.79; H, 4.01; N, 3.98. Found: C, 64.67: H, 3.98; N, 3.69 .

(2E)-1-(4-Bromo-1-naphthyl)-3-(4-aminophenyl)-2-propen-1-one (3). Yield: 94\%, m.p.81$82^{\circ} \mathrm{C}$; IR $\left(\mathrm{KBr}, \mathrm{cm}^{-1}\right): v=1669(\mathrm{CO}$ s-cis $), 1635(\mathrm{CO}$ s-trans $), 991(\mathrm{CH}=\mathrm{CH}), 3576\left(-\mathrm{NH}_{2}\right) .{ }^{1} \mathrm{H}-$ 
NMR $\left(\mathrm{CDCl}_{3}, \mathrm{ppm}\right): \delta=8.15(\mathrm{~d}, 1 \mathrm{H} \alpha), 8.23(\mathrm{~d}, 1 \mathrm{H} \beta), 7.30-7.79(\mathrm{~m}, 10 \mathrm{H} \mathrm{Ar}-\mathrm{H}), 4.06(\mathrm{~s}, 2 \mathrm{H}-$ $\left.\mathrm{NH}_{2}\right) .{ }^{13} \mathrm{C} \mathrm{NMR}\left(\mathrm{CDCl}_{3}, \mathrm{ppm}\right): \delta=118.81\left(\mathrm{C}_{\alpha}\right) 141.81\left(\mathrm{C}_{\beta}\right), 191.34(\mathrm{CO}), 137.99\left(\mathrm{C}_{1}\right), 126.82$ $\left(\mathrm{C}_{2}\right), 131.50\left(\mathrm{C}_{3}\right), 128.60\left(\mathrm{C}_{4}\right), 132.41\left(\mathrm{C}_{5}\right), 126.84\left(\mathrm{C}_{6}\right), 133.10\left(\mathrm{C}_{7}\right), 124.02\left(\mathrm{C}_{8}\right), 132.00\left(\mathrm{C}_{4 \mathrm{a}}\right)$, $132.00\left(\mathrm{C}_{8 \mathrm{a}}\right), 129.42\left(\mathrm{C}_{1}^{\prime}\right), 130.48\left(\mathrm{C}_{2}^{\prime}, \mathrm{C}_{6}{ }^{\prime}\right), 117.42\left(\mathrm{C}_{3}^{\prime}, \mathrm{C}_{5}{ }^{\prime}\right), 135.41\left(\mathrm{C}_{4}{ }^{\prime}\right)$. Anal. Calcd for $\mathrm{C}_{19} \mathrm{H}_{14} \mathrm{BrNO}$ (337.20): C, 64.79; H, 4.01; N, 3.98. Found: C, 64.75; H, 3.92; N, 3.88.

(2E)-1-(4-Bromo-1-naphthyl)-3-(3-bromophenyl)-2-propen-1-one (4) Yield: 95\%, m.p.107$108^{\circ} \mathrm{C}$; IR $\left(\mathrm{KBr}, \mathrm{cm}^{-1}\right): v=1681$ (CO s-cis), 1658 (CO s-trans), $1025(\mathrm{CH}=\mathrm{CH}) .{ }^{1} \mathrm{H}$ NMR $\left(\mathrm{CDCl}_{3}, \mathrm{ppm}\right): \delta=8.31(\mathrm{~d}, 1 \mathrm{H} \alpha), 8.34(\mathrm{~d}, 1 \mathrm{H} \beta), 7.51-8.05(\mathrm{~m}, 10 \mathrm{H} \mathrm{Ar}-\mathrm{H}) .{ }^{13} \mathrm{C} \mathrm{NMR}\left(\mathrm{CDCl}_{3}\right.$, ppm): $\delta=121.64\left(\mathrm{C}_{\alpha}\right) 142.03\left(\mathrm{C}_{\beta}\right), 192.67(\mathrm{CO}), 137.02\left(\mathrm{C}_{1}\right), 127.74\left(\mathrm{C}_{2}\right), 129.48\left(\mathrm{C}_{3}\right), 123.28$ $\left(\mathrm{C}_{4}\right), 128.75\left(\mathrm{C}_{5}\right), 126.74\left(\mathrm{C}_{6}\right), 128.10\left(\mathrm{C}_{7}\right), 134.69\left(\mathrm{C}_{8}\right), 134.99\left(\mathrm{C}_{4 \mathrm{a}}\right), 130.29\left(\mathrm{C}_{8 \mathrm{a}}\right), 140.44\left(\mathrm{C}_{1}{ }^{\prime}\right)$, $129.63\left(\mathrm{C}_{2}^{\prime}\right), 128.28\left(\mathrm{C}_{3}^{\prime}\right), 131.34\left(\mathrm{C}_{4}^{\prime}\right), 130.29\left(\mathrm{C}_{5}^{\prime}\right), 124.99\left(\mathrm{C}_{6}^{\prime}\right)$. Anal. Calcd. for $\mathrm{C}_{19} \mathrm{H}_{12} \mathrm{Br}_{2} \mathrm{O}$ (416.10): C, 54.84; H, 2.91. Found: C, 54.79; H, 2.83.

(2E)-1-(4-Bromo-1-naphthyl)-3-(3-chlorophenyl)-2-propen-1-one (5). Yield: 96\%, m.p.110$111^{\circ} \mathrm{C}$; IR $\left(\mathrm{KBr}, \mathrm{cm}^{-1}\right): v=1686$ (CO s-cis), 1654 (CO s-trans), $1020(\mathrm{CH}=\mathrm{CH}) .{ }^{1} \mathrm{H}$ NMR $\left(\mathrm{CDCl}_{3}, \mathrm{ppm}\right): \delta=8.26(\mathrm{~d}, 1 \mathrm{H} \alpha), 8.28(\mathrm{~d}, 1 \mathrm{H} \beta), 7.47-7.86(\mathrm{~m}, 10 \mathrm{H} \mathrm{Ar}-\mathrm{H}) .{ }^{13} \mathrm{C} \mathrm{NMR}\left(\mathrm{CDCl}_{3}\right.$, ppm): $\delta=122.23\left(\mathrm{C}_{\alpha}\right) 144.65\left(\mathrm{C}_{\beta}\right), 190.18(\mathrm{CO}), 135.61\left(\mathrm{C}_{1}\right), 129.47\left(\mathrm{C}_{2}\right), 132.60\left(\mathrm{C}_{3}\right), 128.93$ $\left(\mathrm{C}_{4}\right), 130.45\left(\mathrm{C}_{5}\right), 128.30\left(\mathrm{C}_{6}\right), 135.04\left(\mathrm{C}_{7}\right), 122.27\left(\mathrm{C}_{8}\right), 130.45\left(\mathrm{C}_{4 \mathrm{a}}\right), 130.66\left(\mathrm{C}_{8 \mathrm{a}}\right), 144.65\left(\mathrm{C}_{1}{ }^{\prime}\right)$, $127.78\left(\mathrm{C}_{2}^{\prime}\right), 135.04\left(\mathrm{C}_{3}^{\prime}\right), 135.49\left(\mathrm{C}_{4}^{\prime}\right), 129.47\left(\mathrm{C}_{5}^{\prime}\right), 124.96\left(\mathrm{C}_{6}^{\prime}\right)$. Anal. Calcd for $\mathrm{C}_{19} \mathrm{H}_{12} \mathrm{BrClO}$ (371.65): C, 61.40; H, 3.25. Found: C, 61.38; H, 3.20.

(2E)-1-(4-Bromo-1-naphthyl)-3-(4-chlorophenyl)-2-propen-1-one (6). Yield: 96\%, m.p.100$101^{\circ} \mathrm{C}$; IR $\left(\mathrm{KBr}, \mathrm{cm}^{-1}\right): v=1679$ (CO s-cis), 1652 (CO s-trans), $1029(\mathrm{CH}=\mathrm{CH}) .{ }^{1} \mathrm{H}$ NMR $\left(\mathrm{CDCl}_{3}, \mathrm{ppm}\right): \delta=8.300(\mathrm{~d}, 1 \mathrm{H} \alpha), 8.32(\mathrm{~d}, 1 \mathrm{H} \beta), 7.39-7.82(\mathrm{~m}, 10 \mathrm{H} \mathrm{Ar}-\mathrm{H}) .{ }^{13} \mathrm{C} \mathrm{NMR}\left(\mathrm{CDCl}_{3}\right.$, ppm): $\delta=121.31\left(\mathrm{C}_{\alpha}\right) 143.72\left(\mathrm{C}_{\beta}\right), 186.60(\mathrm{CO}), 136.858\left(\mathrm{C}_{1}\right), 129.66\left(\mathrm{C}_{2}\right), 128.77\left(\mathrm{C}_{3}\right), 126.86$ $\left(\mathrm{C}_{4}\right), 128.25\left(\mathrm{C}_{5}\right), 126.06\left(\mathrm{C}_{6}\right), 132.89\left(\mathrm{C}_{7}\right), 121.30\left(\mathrm{C}_{8}\right), 130.33\left(\mathrm{C}_{4 \mathrm{a}}\right), 130.77\left(\mathrm{C}_{8 \mathrm{a}}\right), 136.86$ $\left(\mathrm{C}_{1}{ }^{\prime}\right), 127.96\left(\mathrm{C}_{2}^{\prime}, \mathrm{C}_{6}^{\prime}\right), 129.33\left(\mathrm{C}_{3}^{\prime}, \mathrm{C}_{5}^{\prime}\right), 136.89\left(\mathrm{C}_{4}^{\prime}\right)$. Anal. Calcd for $\mathrm{C}_{19} \mathrm{H}_{12} \mathrm{BrClO}$ (371.05): $\mathrm{C}$, 61.40; H, 3.25. Found: C, 61.32; H, 3.22.

(2E)-1-(4-Bromo-1-naphthyl)-3-(4-dimethylaminophenyl)-2-propen-1-one (7). Yield: 93\%, m.p.118-119 ${ }^{\circ}$; IR $\left(\mathrm{KBr}, \mathrm{cm}^{-1}\right): v=1672$ (CO s-cis), 1631 (CO s-trans), $976(\mathrm{CH}=\mathrm{CH}), 3576(-$ $\left.\mathrm{NH}_{2}\right) .{ }^{1} \mathrm{H} \mathrm{NMR}\left(\mathrm{CDCl}_{3}, \mathrm{ppm}\right): \delta=8.12(\mathrm{~d}, 1 \mathrm{H} \alpha), 8.20(\mathrm{~d}, 1 \mathrm{H} \beta), 7.63-8.32(\mathrm{~m}, 10 \mathrm{H} \mathrm{Ar}-\mathrm{H}), 2.88$ $\left.\left(\mathrm{s}, 6 \mathrm{H},\left(\mathrm{CH}_{3}\right)_{2}\right) .{ }^{13} \mathrm{C} \mathrm{NMR}\left(\mathrm{CDCl}_{3}, \mathrm{ppm}\right): \delta=118.80\left(\mathrm{C}_{\alpha}\right) 141.30 \mathrm{C}_{\beta}\right), 190.20(\mathrm{CO}), 42.84\left(\mathrm{CH}_{3}\right)$, $135.00\left(\mathrm{C}_{1}\right), 129.72\left(\mathrm{C}_{2}\right), 127.99\left(\mathrm{C}_{3}\right), 129.09\left(\mathrm{C}_{4}\right), 130.14\left(\mathrm{C}_{5}\right), 128.95\left(\mathrm{C}_{6}\right), 128.53\left(\mathrm{C}_{7}\right), 124.72$ $\left(\mathrm{C}_{8}\right), 130.15\left(\mathrm{C}_{4 \mathrm{a}}\right), 129.09\left(\mathrm{C}_{8 \mathrm{a}}\right), 124.72\left(\mathrm{C}_{1}^{\prime}\right), 126.92\left(\mathrm{C}_{2}^{\prime}, \mathrm{C}_{6}^{\prime}\right), 114.42\left(\mathrm{C}_{3}^{\prime}, \mathrm{C}_{5}^{\prime}\right), 152.47\left(\mathrm{C}_{4}^{\prime}\right)$. Anal. Calcd for $\mathrm{C}_{21} \mathrm{H}_{18} \mathrm{BrNO}$ (380.27): C, 66.33; H, 4.77; N, 3.68. Found: C, 66.34; H, 4.67; N, 3.64 .

(2E)-1-(4-Bromo-1-naphthyl)-3-(4-hydroxyphenyl)-2-propen-1-one (8). Yield: 91\%, m.p.97-98 ${ }^{\circ}$; IR (KBr, cm $\left.{ }^{-1}\right): v=1671$ (CO s-cis), 1635 (CO s-trans), $1015(\mathrm{CH}=\mathrm{CH}), 3565$ ($\mathrm{OH}) .{ }^{1} \mathrm{H}$ NMR $\left(\mathrm{CDCl}_{3}, \mathrm{ppm}\right): \delta=8.18(\mathrm{~d}, 1 \mathrm{H} \alpha), 8.20(\mathrm{~d}, 1 \mathrm{H} \beta), 7.69-7.84(\mathrm{~m}, 10 \mathrm{H} \mathrm{Ar}-\mathrm{H}), 4.75$ (s, $1 \mathrm{H}-\mathrm{OH}) .{ }^{13} \mathrm{C}-\mathrm{NMR}\left(\mathrm{CDCl}_{3}, \mathrm{ppm}\right): \delta=119.72\left(\mathrm{C}_{\alpha}\right) 143.56\left(\mathrm{C}_{\beta}\right), 191.20(\mathrm{CO}), 134.52\left(\mathrm{C}_{1}\right)$, $128.53\left(\mathrm{C}_{2}\right), 130.05\left(\mathrm{C}_{3}\right), 128.53\left(\mathrm{C}_{4}\right), 130.30\left(\mathrm{C}_{5}\right), 128.34\left(\mathrm{C}_{6}\right), 132.14\left(\mathrm{C}_{7}\right), 124.92\left(\mathrm{C}_{8}\right), 132.14$ 
$\left(\mathrm{C}_{4 \mathrm{a}}\right), 130.02\left(\mathrm{C}_{8 \mathrm{a}}\right), 130.03\left(\mathrm{C}_{1}{ }^{\prime}\right), 129.46\left(\mathrm{C}_{2}^{\prime}, \mathrm{C}_{6}{ }^{\prime}\right), 116.99\left(\mathrm{C}_{3}{ }^{\prime}, \mathrm{C}_{5}{ }^{\prime}\right), 157.92\left(\mathrm{C}_{4}{ }^{\prime}\right)$. Anal. Calcd. for $\mathrm{C}_{19} \mathrm{H}_{13} \mathrm{BrO}_{2}$ (353.20): C, 64.61; H, 3.71. Found: C, 64.59; H, 3.69.

(2E)-1-(4-Bromo-1-naphthyl)-3-(4-methoxyphenyl)-2-propen-1-one (9). Yield: 96\%, m.p.123-124 ${ }^{\circ} \mathrm{C}$; IR (KBr, cm $\left.{ }^{-1}\right): v=1685$ (CO s-cis), 1641 (CO s-trans), $1024(\mathrm{CH}=\mathrm{CH}) .{ }^{1} \mathrm{H}$ $\operatorname{NMR}\left(\mathrm{CDCl}_{3}, \mathrm{ppm}\right): \delta=8.21(\mathrm{~d}, 1 \mathrm{H} \alpha), 8.34(\mathrm{~d}, 1 \mathrm{H} \beta), 7.05-7.82(\mathrm{~m}, 10 \mathrm{H} \mathrm{Ar}-\mathrm{H}) 4.13(\mathrm{~s}, 3 \mathrm{H}-$ $\left.\mathrm{OCH}_{3}\right) .{ }^{13} \mathrm{C} \mathrm{NMR}\left(\mathrm{CDCl}_{3}, \mathrm{ppm}\right) \delta=121.44\left(\mathrm{C}_{\alpha}\right) 142.62\left(\mathrm{C}_{\beta}\right), 193.90(\mathrm{CO}), 53.81\left(-\mathrm{OCH}_{3}\right)$ $138.21\left(\mathrm{C}_{1}\right), 129.90\left(\mathrm{C}_{2}\right), 128.85\left(\mathrm{C}_{3}\right), 121.96\left(\mathrm{C}_{4}\right), 128.85\left(\mathrm{C}_{5}\right), 126.70\left(\mathrm{C}_{6}\right), 127.10\left(\mathrm{C}_{7}\right), 128.17$ $\left(\mathrm{C}_{8}\right), 132.22\left(\mathrm{C}_{4 \mathrm{a}}\right), 130.60\left(\mathrm{C}_{8 \mathrm{a}}\right), 131.75\left(\mathrm{C}_{1}^{\prime}\right), 127.10\left(\mathrm{C}_{2}^{\prime}, \mathrm{C}_{6}{ }^{\prime}\right), 116.99\left(\mathrm{C}_{3}{ }^{\prime}, \mathrm{C}_{5}{ }^{\prime}\right), 163.45\left(\mathrm{C}_{4}{ }^{\prime}\right)$. Anal. Calcd for $\mathrm{C}_{20} \mathrm{H}_{15} \mathrm{BrO}_{2}$ (367.23): C, 65.40; H, 4.12. Found: C, 65.39; H, 4.00.

(2E)-1-(4-Bromo-1-naphthyl)-3-(4-methylphenyl)-2-propen-1-one (10). Yield: 94\%, m.p.95$96^{\circ} \mathrm{C}$; IR $\left(\mathrm{KBr}, \mathrm{cm}^{-1}\right): v=1676(\mathrm{CO}$ s-cis $), 1642$ (CO s-trans), $993(\mathrm{CH}=\mathrm{CH}) .{ }^{1} \mathrm{H}$ NMR $\left(\mathrm{CDCl}_{3}\right.$, ppm): $\delta=8.25(\mathrm{~d}, 1 \mathrm{H} \alpha), 8.36(\mathrm{~d}, 1 \mathrm{H} \beta), 7.21-7.85(\mathrm{~m}, 10 \mathrm{H} \mathrm{Ar}-\mathrm{H}) 2.57\left(\mathrm{~s}, 3 \mathrm{H}-\mathrm{CH}_{3}\right) .{ }^{13} \mathrm{C} \mathrm{NMR}$ $\left(\mathrm{CDCl}_{3}, \mathrm{ppm}\right): \delta=120.48\left(\mathrm{C}_{\alpha}\right) 143.16\left(\mathrm{C}_{\beta}\right), 191.99(\mathrm{CO}), 25.30\left(-\mathrm{CH}_{3}\right) 137.25\left(\mathrm{C}_{1}\right), 129.79\left(\mathrm{C}_{2}\right)$, $131.09\left(\mathrm{C}_{3}\right), 128.80\left(\mathrm{C}_{4}\right), 130.21\left(\mathrm{C}_{5}\right), 128.61\left(\mathrm{C}_{6}\right), 132.29\left(\mathrm{C}_{7}\right), 122.61\left(\mathrm{C}_{8}\right), 130.21\left(\mathrm{C}_{4 \mathrm{a}}\right)$, $130.22\left(\mathrm{C}_{8 \mathrm{a}}\right), 137.25\left(\mathrm{C}_{1}^{\prime}\right), 126.43\left(\mathrm{C}_{2}^{\prime}, \mathrm{C}_{6}{ }^{\prime}\right), 129.79\left(\mathrm{C}_{3}^{\prime}, \mathrm{C}_{5}^{\prime}\right)$, $137.25\left(\mathrm{C}_{4}{ }^{\prime}\right)$. Anal. Calcd for $\mathrm{C}_{20} \mathrm{H}_{15} \mathrm{BrO}$ (351.23): C, 68.39; H, 4.30. Found: C, 68.34: H, 3.99.

(2E)-1-(4-Bromo-1-naphthyl)-3-(2-nitrophenyl)-2-propen-1-one (11). Yield: 91\%, m.p.76$77^{\circ} \mathrm{C}$; IR $\left(\mathrm{KBr}, \mathrm{cm}^{-1}\right): v=1685$ (CO s-cis), 1669 (CO s-trans), $1023(\mathrm{CH}=\mathrm{CH}) .{ }^{1} \mathrm{H}$ NMR $\left(\mathrm{CDCl}_{3}, \mathrm{ppm}\right): \delta=8.27(\mathrm{~d}, 1 \mathrm{H} \alpha), 8.39(\mathrm{~d}, 1 \mathrm{H} \beta), 6.88-8.17(\mathrm{~m}, 10 \mathrm{H} \mathrm{Ar}-\mathrm{H}) .{ }^{13} \mathrm{C} \mathrm{NMR}\left(\mathrm{CDCl}_{3}\right.$, ppm): $\delta=122.17\left(\mathrm{C}_{\alpha}\right)$ 146.16( $\left.\mathrm{C}_{\beta}\right), 194.21(\mathrm{CO}), 138.64\left(\mathrm{C}_{1}\right), 129.80\left(\mathrm{C}_{2}\right), 128.70\left(\mathrm{C}_{3}\right), 122.17\left(\mathrm{C}_{4}\right)$, $128.70\left(\mathrm{C}_{5}\right), 126.90\left(\mathrm{C}_{6}\right), 133.89\left(\mathrm{C}_{7}\right), 122.17\left(\mathrm{C}_{8}\right), 130.92\left(\mathrm{C}_{4 \mathrm{a}}\right), 131.31\left(\mathrm{C}_{8 \mathrm{a}}\right), 133.89\left(\mathrm{C}_{1}{ }^{\prime}\right)$, $46.64\left(\mathrm{C}_{2}^{\prime}\right), 124.13\left(\mathrm{C}_{3}^{\prime}\right), 128.70\left(\mathrm{C}_{4}^{\prime}\right), 133.89\left(\mathrm{C}_{5}^{\prime}\right), 127.58\left(\mathrm{C}_{6}^{\prime}\right)$. Anal. Calcd for $\mathrm{C}_{19} \mathrm{H}_{12} \mathrm{BrNO}_{3}$ (382.20): C, 59.71; H, 3.16; N, 3.66. Found: C, 59.65; H, 3.08; N, 3.56.

(2E)-1-(4-Bromo-1-naphthyl)-3-(3-nitrophenyl)-2-propen-1-one (12). Yield: 94\%, m.p.88$89^{\circ} \mathrm{C}$; IR $\left(\mathrm{KBr}, \mathrm{cm}^{-1}\right): v=1678(\mathrm{CO}$ s-cis $), 1663$ (CO s-trans), $1017(\mathrm{CH}=\mathrm{CH}) .{ }^{1} \mathrm{H} \mathrm{NMR}\left(\mathrm{CDCl}_{3}\right.$, ppm): $\delta=8.31(\mathrm{~d}, 1 \mathrm{H} \alpha), 8.38(\mathrm{~d}, 1 \mathrm{H} \beta), 7.37-8.43(\mathrm{~m}, 10 \mathrm{H} \mathrm{Ar}-\mathrm{H}) .{ }^{13} \mathrm{C} \mathrm{NMR}\left(\mathrm{CDCl}_{3}, \mathrm{ppm}\right): \delta=$ $122.72\left(\mathrm{C}_{\alpha}\right) 145.39\left(\mathrm{C}_{\beta}\right), 193.84(\mathrm{CO}), 138.36\left(\mathrm{C}_{1}\right), 128.79\left(\mathrm{C}_{2}\right), 130.09\left(\mathrm{C}_{3}\right), 128.72\left(\mathrm{C}_{4}\right)$, $127.38\left(\mathrm{C}_{5}\right), 128.49\left(\mathrm{C}_{6}\right), 133.65\left(\mathrm{C}_{7}\right), 122.74\left(\mathrm{C}_{8}\right), 130.03\left(\mathrm{C}_{4 \mathrm{a}}\right), 130.09\left(\mathrm{C}_{8 \mathrm{a}}\right), 134.03\left(\mathrm{C}_{1}{ }^{\prime}\right)$, $112.72\left(\mathrm{C}_{2}^{\prime}\right), 148.76\left(\mathrm{C}_{3}^{\prime}\right), 114.02\left(\mathrm{C}_{4}^{\prime}\right), 126.09\left(\mathrm{C}_{5}^{\prime}\right), 122.39\left(\mathrm{C}_{6}^{\prime}\right)$. Anal. Calcd for $\mathrm{C}_{19} \mathrm{H}_{12} \mathrm{BrNO}_{3}$ (382.20): C, 59.71; H, 3.16; N, 3.66. Found: C, 59.68; H, 3.11; N, 3.58.

(2E)-1-(4-Bromo-1-naphthyl)-3-(4-nitrophenyl)-2-propen-1-one. (13) Yield: 94\%,

m.p. 116-117 ${ }^{\circ}$; IR $\left(\mathrm{KBr}, \mathrm{cm}^{-1}\right): v=1684$ (CO s-cis), $1672(\mathrm{CO}$ s-trans $), 1016(\mathrm{CH}=\mathrm{CH}) .{ }^{1} \mathrm{H}$ NMR $\left(\mathrm{CDCl}_{3}, \mathrm{ppm}\right): \delta=8.33(\mathrm{~d}, 1 \mathrm{H} \alpha), 8.40(\mathrm{~d}, 1 \mathrm{H} \beta), 7.35-8.13(\mathrm{~m}, 10 \mathrm{H} \mathrm{Ar}-\mathrm{H}) .{ }^{13} \mathrm{C}-$ $\operatorname{NMR}\left(\mathrm{CDCl}_{3}, \mathrm{ppm}\right): \delta=123.43\left(\mathrm{C}_{\alpha}\right) 145.18\left(\mathrm{C}_{\beta}\right), 193.552(\mathrm{CO}), 139.10\left(\mathrm{C}_{1}\right), 129.58\left(\mathrm{C}_{2}\right), 132.10$ $\left(\mathrm{C}_{3}\right), 128.55\left(\mathrm{C}_{4}\right), 129.58\left(\mathrm{C}_{5}\right), 127.20\left(\mathrm{C}_{6}\right), 127.20\left(\mathrm{C}_{7}\right), 129.58\left(\mathrm{C}_{8}\right), 135.92\left(\mathrm{C}_{4 \mathrm{a}}\right), 132.10\left(\mathrm{C}_{8 \mathrm{a}}\right)$, $142.47\left(\mathrm{C}_{1}^{\prime}\right), 127.60\left(\mathrm{C}_{2}^{\prime}, \mathrm{C}_{6}^{\prime}\right), 115.72\left(\mathrm{C}_{3}^{\prime}, \mathrm{C}_{5}^{\prime}\right)$, 148.50( $\left.\mathrm{C}_{4}^{\prime}\right)$. Anal. Calcd for $\mathrm{C}_{19} \mathrm{H}_{12} \mathrm{BrNO}_{3}$ (382.20): C, 59.71; H, 3.16; N, 3.66. Found: C, 59.68; H, 3.11; N, 3.58. 


\section{Conclusions}

This method is a very efficient and selective protocol for crossed-aldol condensation of 4-bromo1-naphthyl ketones and aldehydes to produce high yields of 4-bromo-1-naphthyl chalcones in the presence of a reusable and environmentally beginning catalyst silica-sulfuric acid. Operative simplicity, easy work-up procedure, better yield including washing the mixture followed by evaporation of the solvent are other advantages of this method.

\section{Acknowledgements}

The authors thank Dr. D.D. Sung, South Korea, for financial support and for recording NMR Spectra of all compounds, and Prof. Dr. P. Ananthakrishna Nadar, Secretary of the International Group of Correlation Analysis in Chemistry, Currently Emeritus Scientist, Faculty of Chemical Sciences, Bharath Institute of Technology, (Deemed University), Chennai, for NMR data analysis.

\section{References}

1. (a) Tanaka, K.; Toda, F. Chem. Rev. 2000, 100, 1025. (b) Cave, G. W. V.; Taston, C. L.; Scott, J. L. Chem. Commun. 2001, 2159.

2. (a) John, E. J.; Mathew, S. B. J Chem. Educ. 2003, 80, 1446. (b) Misra, S. S.; Tewari, R. S.; Nath, B. Indian J. Appl. Chem. 1971, 34, 260.

3. Venkat Reddy, G.; Maitraie, D.; Narasiah, B.; Rambabu, Y.; Shanthan Rao, D. Synth Commun. 2001, 3, 2884.

4. (a) Guthrie, W.; Can J. Chem. 1991, 69, 339. (b) Guthrie, W. J. Am. Chem. Soc. 1991, 109, 6609. (c) Nielson, A.T.; Houlihan, W.J.; Org React. 1968, 16, 1.

5. (a) Sung, D. D.; Ananthakrishna Nadar, P. Indian J. Chem. 2000, 39(A), 1066. (b) Sung, D. D.; Ananthakrishna Nadar, P. Bull. Korean Chem. Soc. 1999, 20, 1487.

6. (a) Smith, M. B.; March, J. Advanced Organic Chemistry, Reaction, Mechanism \& Structure, Wiley: New York, 2001, pp 1218-1224. (b) Norcross, R. D.; Peterson, L. Chem. Rev. 1995, 95, 2041. (c) Trost, B. M.; Fleming, L. Comprehensive Organic Synthesis, Pergamon: Oxford, 1991, Vol.2, Parts 1.4-1.7.

7. Deli, J.; Lorand, T.; Szabo, D.; Foldesi, A. Pharmaz. 1984, 39, 539.

8. Winternitz, F.; Conder, G.; Vassort, P.; Tetrahedron Lett. 1986, $26,5981$.

9. (a) Misra, S. S.; Tewari, R. S. J Indian Chem. Soc. 1973, 50, 68. (b) Lafen, L.; Ger. Pp 2010180, 1970; Chem. Abstr 1970, 73, 120342 s.

10. Hathaway, B. A. J. Chem. Educ. 1987, 64, 367. 
11. Nakano, T.; Irifune, S.; Umano, S.; Inada, A.; Ishii, Y.; Ogawa, M. J. Org. Chem. 1987, 52, 2239.

12. Irie, K.; Watanabe, K. Bull. Chem. Soc. Jpn. 1980, 52, 1366.

13. Zheng Wang, L.; Shao, J.; Zhong, Q. Synth. Commun. 1987, 27, 351.

14. Yadav, J. S.; Subba Reddy, B. V.; Nagaraju, Sarma, J.A.R.P. Synth. Commun. 2002, 32, 893.

15. Iranpoor, N.; Zeynizadeli, B.; Aghapour, A.; J. Chem. Res. (S). 1999, 554.

16. (a) Salehi, P.; Khodaei, M. M.; Zolfigol, M. A.; Keyvan, A. Monatsh. Chem. 2002, 133, 1291. (b) Deng, G. S.; Ren, T. G. Synth. Commun. 2003, 33, 2995.

17. Kalluraya, B.; Ray, G.; Indian J. Chem. 2003, 42(B), 2556.

18. Zolfigol, M. A.; Tetrahedron 2001, 57, 9509.

19. Watanabe, K.; Imazawa, A. Bull. Chem. Soc. Jpn. 1982, 55, 3208.

20. Hays, W. P.; Timmons, C. J.; Spectrochim Acta 1968, 24A, 3239.

21. Misra, L.; Singh, A. K. Indian J. Chem. 2001, 40A, 1288.

22. Hamer, G. K.; Peat, I. R.; Reynolds, W. F. Can. J. Chem. 1973, 51, 915.

23. Dhami, K. S.; Stothers, J. B. Can. J. Chem. 1965, 43, 510.

24. Wells, P. R.; Arnoid, D. P. J. Chem. Soc., Perkins Trans 2 1970, 1745.

25. Doddrell, D.; Wells, P. R. J. Chem. Soc, Perkins Trans 2 1973, 1333.

26. Johnson, L. F.; Jonkowski, W. C. Carbon-13 NMR Spectra, Wiley-Interscience: New York, 1972.

27. Kitching, W.; Bullpitt, M.; Doddrell, D.; Adcock, W. Org. Magnetic Resonance 1974, 6, 289. 